Cahiers de littérature orale

$70 \mid 2011$

L'adresse indirecte ou la parole détournée

\title{
L'art de dire sans dire en Kabylie
}

\section{Tassadit Yacine}

\section{(2) OpenEdition}

Journals

Édition électronique

URL : https://journals.openedition.org/clo/1271

DOI : 10.4000/clo.1271

ISSN : 2266-1816

Éditeur

INALCO

\section{Édition imprimée}

Date de publication : 30 décembre 2011

Pagination : 67-86

ISBN : 978-2-85831-202-3

ISSN : 0396-891X

\section{Référence électronique}

Tassadit Yacine, "L'art de dire sans dire en Kabylie », Cahiers de littérature orale [En ligne], 70 | 2011, mis en ligne le 17 mars 2013, consulté le 30 juin 2021. URL : http://journals.openedition.org/clo/1271 ; DOI : https://doi.org/10.4000/clo.1271

Ce document a été généré automatiquement le 30 juin 2021

\section{(c) (†) 8)}

Cahiers de littérature orale est mis à disposition selon les termes de la Licence Creative Commons Attribution - Pas d'Utilisation Commerciale 4.0 International. 


\title{
L'art de dire sans dire en Kabylie
}

\author{
Tassadit Yacine
}

Il n'est pas bon de s'incliner

Pas bon de se taire [...]

Cheikh Mohand

\section{Une approche complexe}

1 La Kabylie est une région montagneuse située à cent trente kilomètres d'Alger qui a conservé, plus qu'ailleurs, des pratiques langagières (une tradition orale ancienne) et rituelles. En raison d'un mode de vie presque en autarcie, l'héritage culturel ancien a été préservé jusqu'à la colonisation française (1830) dont les effets ont permis une pénétration culturelle et un changement social accéléré. Ce phénomène a agi sur les modes anciens de création, de transmission et de perpétuation de la culture menacée de perte au profit d'une homogénéisation progressive des pratiques culturelles et langagières.

2 Cette position topographique a, en outre, favorisé une diversité culturelle qui attira à partir de la fin du xix ${ }^{e}$ siècle un nombre important d'ethnologues.

3 Le code social et culturel kabyle accorde une place importante au discours et aux différentes formes d'énonciation dont les modes d'expression peuvent être directs et indirects. La parole joue ainsi un rôle social important selon le statut social, l'âge et l'appartenance sexuelle. À côté de la maîtrise des savoirs locaux (poésie, mythologie, généalogie, droit coutumier, contes, légendes, récits de fondation), la détention des codes langagiers est tout particulièrement valorisée, elle est perçue comme une véritable performance. Dans certaines régions de Kabylie, il est en outre commun d'envoyer le fils aîné apprendre l'art du verbe chez des maîtres connus sous le nom de forgerons de la langue (aheddad bbwawal). Ce sont des hommes, adultes, qui manient les tournures, les doubles sens, les sens cachés ainsi que les différents codes de la langue ${ }^{1}$. La parole obéit à une hiérarchie de valeurs où les initiés (amousnaw-s : savants et sages à la fois) occupent la position la plus élevée dans l'échelle sociale, viennent ensuite les poètes (ceux qui ont l'art de délier, de dénouer, d'élucider, de pénétrer le sens profond 
de la langue d'où le terme significatif d'asefrou pour désigner le poème. Le code langagier établit une hiérarchie entre les grands poètes (ifsihen) distinguant les meddah$s$ (poètes ambulants), les berrah-s (hérauts), etc. (Bourdieu, 1980 ; Mammeri, 1978).

4 Il est, en effet, socialement admis de recourir à cette façon de voir, de penser et de s'exprimer qui exige de son interlocuteur une attention et anticipation dans les situations les plus diverses et les plus variées. L'adresse indirecte est à la fois crainte (car elle renferme de véritables pièges) et prisée en ce qu'elle permet de révéler la culture et l'intelligence de l'interlocuteur face à son partenaire et de jauger son niveau. Le discours kabyle distingue ainsi l'adresse directe de l'adresse indirecte. Si la première va de soi et n'est pas nommée dans la langue, la seconde en revanche est définie par plusieurs termes dont le plus connu est lemâun forme pluriel de lmaâna (de l'arabe sens). En kabyle, ce terme a certes la même signification, mais il renvoie aussi à l'éducation et, par-delà, à toute personne encline à la réplique. Cette même pratique fondée sur le jeu du langage se retrouve également au Maroc chez les Chleuhs. Elle prend appui sur l'implicite comme dynamique de construction de sens et comme principe de création et de spéculation renvoyant à une compétence linguistique. L'on sait également que le sens patent se fonde sur un non-dit et sur la capacité de reconnaître et de jouer sur les structures de la langue.

Cette façon de procéder est en réalité une manière de dire que toute pensée n'est pas « dicible » ou bien que la richesse et la diversité de l'intellect ne sont pas réductibles aux seuls mots ni à leur sens originel. Il faut comprendre qu'il existe un code langagier qui dépasse la langue et un sens qui transcende le sens supposé. Le signifiant serait ainsi pauvre devant l'infini de l'idée et de l'éventail de ses nuances. C'est d'ailleurs sur cette richesse que jouent les mystiques qui entendent s'exprimer uniquement pour les initiés ${ }^{2}$.

[...] la pensée non formulée verbalement, non nommée, non identifiée fournit un aliment continuel à la prospection mentale, à l'activité spéculative. Il nous apprend aussi qu'à partir de l'instant où la pensée est verbalisée, nommée elle perd tout caractère dynamique. Elle devient alors un objet statufié, un cliché figé. Mentalement inerte et bon à mémoriser et à classer.

(Jouad, 1989, 158)

6 Pourquoi recourir à l'adresse indirecte lorsque l'on peut communiquer directement ? N'y aurait-il pas dans ce mode d'expression un rôle important qui échappe à l'intelligibilité du sens commun pour revêtir d'autres fonctions là où on les suspecte le moins?

7 Ce qui semble un détour, un jeu, une forme sournoise d'expression cache sans doute une richesse dans les procédés liés au langage que les locuteurs tendent à perdre parce qu'ils ont perdu le code. La connaissance des modes d'expression de la société kabyle «traditionnelle » permet de mieux comprendre la place de la parole et les différentes adresses (directe et indirecte) auxquelles sont soumis les agents sociaux. Dans la Kabylie d'autrefois, les modes d'expression obéissaient à des règles strictes pour les hommes, les femmes, les religieux, les bergers, les esclaves. Il n'en est pas de même dans les sociétés qui ont subi des transformations drastiques et notamment en ce qui concerne la gestion de la parole. Jadis expertes dans l'art de l'indirect, parce que les femmes n'avaient droit à aucun autre mode d'expression, qu'en est-il aujourd'hui ? S'expriment-elles de façon directe ou bien ont-elles adopté un mode d'expression différent bien que toujours indirect? Quelles voies (ou quelles voix) ont-elles empruntées? 
Outre les formes orales de production de ce moyen d'expression dans une société traditionnelle comme la Kabylie - où la parole avait un rôle très important comme l'a souligné Mouloud Mammeri dans Poèmes kabyles anciens (1980) - nous tenterons de montrer dans cet article la portée de l'adresse indirecte dans différents espaces d'expression: dans la vie quotidienne, mais aussi dans la prophétie (celle de Cheikh Mohand), comme dans l'univers de la poésie chantée; autre moyen d'éviter le face-àface avec autrui tout en assénant des vérités indicibles dans des situations ordinaires. Les détours de la langue et les mécanismes adoptés pour parvenir à faire entendre un message sont dignes d'être connus et déconstruits.

\section{Statuts et droit à la parole}

\section{Du masculin et du féminin}

9 L'adresse indirecte participe d'un jeu fondé sur l'implicite et le non-dit qui, cependant, permet «d'accorder» ceux qui sont censés partager des valeurs communes et d'éliminer les autres candidats. Ces derniers sont supposés hors jeu parce qu'ils ne maitrisent pas les enjeux qui en découlent comme dans les relations entre les hommes et les femmes ou dans l'écart qui sépare les catégories sociales et les classes d'âge.

La représentation sociale fait une place capitale aux valeurs masculines fondées sur le fait d'affronter l'autre (le masculin de statut égal) sans tergiverser, contrairement à la manière de procéder des femmes. L'homme est direct, la femme est indirecte ; l'homme est décidé, droit, la femme est tordue (telles la faucille, la louche), hésitante, indécise, imprécise, peu sûre d'elle (Bourdieu, 1980).

11 Dans la vie quotidienne, un homme d'honneur, un vrai (celui qui est du côté de la virtus) doit affronter son adversaire, aller de l'avant et ne jamais hésiter. Ce sont là des qualités qui caractérisent l'argaz socialement reconnu par ses pairs: on dit de lui yetqabal, il affronte, il regarde droit dans les yeux et tranche ses mots (il est commun de le comparer aux ciseaux, ou à la faux). Mais hormis cette capacité d'aller vers le risque de manière frontale, argaz est aussi celui qui a la capacité de décrypter, de décoder un énoncé chargé d'allusions, de paraboles, de métaphores et de non-dits comme on peut les trouver dans la langue quotidienne et en littérature.

12 Le langage permet en effet de comprendre les rapports de force et l'enracinement culturel sous-jacent. Car si le code de l'honneur (nif) est valorisé dans tous les cas parce qu'il met en avant les valeurs dominantes de la masculinité -, il n'en demeure pas moins qu'il y a une marge d'expression différente. Tout en reconnaissant les rapports de force, celle-ci fait mine de les ignorer grâce au mode indirect. Dans la société kabyle - comme dans beaucoup d'autres -, la règle consiste à ne pas blesser autrui, d'où l'évitement des réponses négatives. On répond toujours par l'affirmative en laissant le soin à l'interlocuteur de deviner lui-même le sens des propos de son interlocuteur. L'expression employée de façon pléthorique étant inchallah ou ma yella lmektoub (si c'est écrit). Cette tendance à éviter la négativité (la noirceur, ce qui est de mauvais augure) est très présente dans la culture, elle est inscrite dans les structures au point de faire corps avec l'interlocuteur. 

auprès des Ait Kaci, une grande famille de la région qui avait un pouvoir considérable sur les populations de la plaine au XIX ${ }^{e}$ siècle. Ils avaient pour habitude de prendre pour femmes les plus belles de la région, mais, dès qu'il y avait un différend entre la jeune épousée et sa belle famille, ils avaient recours à un moyen des plus inhumains qui consistait à «suspendre » (aâlleq) la jeune femme pendant sept ans. Cette coutume devait obliger la jeune femme à rester sous la tutelle du mari alors qu'elle était répudiée. Le père de la jeune femme vint solliciter alors l'aide du cheikh en espérant qu'il vienne lui porter secours. Les seigneurs de la région étaient sûrs de leur puissance et de leurs droits, car leurs lois, pensaient-ils, étaient supérieures à celles de tous les villages environnants et personne ne pouvait les remettre en question. Du temps passa et un jour les Aït Kaci, eux-mêmes, eurent à demander conseil au cheikh. Arrivés près de la maison, ils attachèrent leurs chevaux comme à l'accoutumée. Cependant, le lendemain au réveil quand ils voulurent partir, ils trouvèrent les chevaux sellés d'une drôle de manière: la sangle avant tournée vers la croupe et celle de l'arrière vers le poitrail. offusqués, ils s'en allèrent trouver le cheikh qui leur fit cette réplique :

Je croyais que c'était votre manière de seller les chevaux, voulant signifier par là que, quand les Ait Kaci revendiquaient le droit d'avoir une coutume particulière à eux, ils la faisaient à leur avantage, mais il y a des conditions qui sont communes à tout le monde; il n'y a qu'une façon de seller les chevaux, avec la sangle avant vers le poitrail et la sangle arrière vers la croupe, de la même façon il y a une voie juste, valable pour tous.

(Mammeri, 2005, t. II, 41)

Dans un autre exemple, le cheikh est encore plus explicite sur ce qu'est l'adresse indirecte. Voici le résumé de l'histoire. 

le déshéritant. Le cheikh sans le regarder continuait à donner du grain à l'un de ses pigeonneaux :

Pigeonneau, lui dit-il, adresse ta demande à ton créateur pas à ton géniteur !

Frustré, le jeune homme s'en alla et ne tarda pas à revenir à la charge une deuxième puis une troisième fois.

Excédé, le cheikh lui répondit:

" Sot, qu'est-ce que je ne cesse de te répéter depuis tout à l'heure?

Quand je disais au poussin de s'adresser à son créateur, non à son géniteur c'est à

toi que je parlais. Alors va, sors du pays pour gagner ta subsistance. »

(Mammeri, ibid., 124)

Cet exemple montre la force de l'adresse indirecte qui consiste à saisir un discours adressé en principe à un tiers alors qu'il s'adresse à soi. En évoquant cette vérité, le cheikh joue sur un double registre, celui du particulier et de l'universel... L'universel permet au particulier de trouver sens sans avoir à en souffrir. Le fait de savoir que cela ne s'adresse pas uniquement à lui permet de trouver une issue à ses problèmes. La supériorité du cheikh est ici réelle, mais suggérée dès lors que l'adresse indirecte permet de dire sans dire.

Mouloud Mammeri a consacré un ouvrage entier aux dits de Cheikh Mohand où cette manière de procéder, bien qu'exceptionnelle, est en quelque sorte une règle puisqu'elle désigne son détenteur comme hors du commun. Ces paroles sont d'autant plus significatives qu'elles ne s'inscrivent jamais dans le direct et l'explicite. L'interlocuteur supposé doit lui-même interpréter les paroles du cheikh sans qu'elles lui soient adressées. Les Algériens et les Kabyles, en particulier, ont une expression courante pour désigner ce procédé : l'intelligent comprendra (fhem ya lfahem). L'homme avisé se nourrit de l'expérience des autres et sait aussi que l'on ne s'adressera jamais à lui directement et non comme un âne que l'on conduit avec un bâton... Les Aït Kaci n'auraient jamais accepté d'entendre le cheikh (car cela supposait une mise en question du mode de gestion de leur pouvoir) et de tenir compte de son avis s'il n'avait pas soigné son expression et respecté les usages en vigueur. L'adresse indirecte est un mode supérieur d'expression, car il relève de la performance et de l'esthétique. Il est aussi un mode ludique, le jeu ayant pour fonction d'éviter le conflit, le face- à-face, qui mettrait l'interlocuteur dans l'obligation de se confronter à l'émetteur. L'adresse indirecte chez Cheikh Mohand est une parole libre et libérée qui, dans la pratique, ne vise personne. Elle est d'autant plus percutante que son porteur fait mine de reconnaître le pouvoir temporel des Aït Kaci. Aux yeux du groupe elle apparaît comme bien supérieure aux lois des dominants locaux. Ainsi ceux qui viennent lui rendre visite (pour prendre conseil) sont libres de faire leur, ou non, cette parole. Les « dits » de Cheikh Mohand sont, pour certains, devenus proverbes grâce à leur dimension universelle même s'ils sont tirés d'une expérience spécifique (Mammeri, 2005).

\section{Dominés et langage symbolique}

\section{Dans la vie quotidienne}

21 Outre ces discours codés, l'adresse indirecte s'inscrit dans le quotidien, dans des situations ordinaires où l'enjeu vise l'ordre symbolique. Les luttes engagées par les femmes pour sauvegarder une position dans l'espace domestique exigent le recours à 
ce mode indirect d'expression: on dit d'ailleurs qu'elles savent "frapper " avec des sous-entendus (lemâun). Leurs luttes s'organisent autour de cette compétence linguistique et de ce véritable savoir-faire empreint parfois d'esthétique (poésie, rythmique, musicalité). La langue travaillée devient ainsi un moyen, un subterfuge pour dire sans dire, affronter sans donner l'impression qu'on transgresse de manière ouverte.

Ainsi dans cette joute qui oppose deux femmes : « Moi, je sais préparer le dîner », dit la première. Et la seconde de répondre: «Est supérieure celle qui a la science du feu (expression populaire) ». Sans feu, il est évident qu'on ne peut parvenir à la préparation du dîner et celle qui est chargée de ramasser du bois (ici la belle-sœur), a le pouvoir d'annuler le dîner. Le pouvoir est inscrit visiblement dans l'art de manier le verbe ainsi que tout l'implicite qu'il renferme comme l'exprime clairement le proverbe kabyle : bab ggiles medden akw ines (litt. "Celui qui a la langue a pour lui le monde» ou " $\mathrm{A}$ l'éloquence point d'échappatoire »). L'implicite concerne toutes les classes d'âge et tous les moments de la vie, en particulier lorsque l'intimité entre en ligne de compte.

Dominées, les femmes sont tenues d'être respectueuses, de ne pas élever la voix, de ne pas créer de problèmes à l'entourage (belle-mère, belles-sœurs, voisines). Car une maison où les femmes se livrent des batailles régulières est très mal perçue par l'opinion. On dit d'elles qu'elles ne sont pas tenues, qu'elles n'ont pas de mors à l'instar des chevaux. Pour cette raison, le seul mode d'expression qui reste possible consiste à contourner la règle en excellant dans l'art du langage (poétique si possible). On rapporte comment, dans un village de la haute montagne, une mère offensée par ses compagnes - parce qu'elle avait un fils unique -, sut se tirer magistralement d'affaire en usant des mêmes procédés que ceux utilisés par celles qui l'avaient provoquée. Voici le contenu du chant des femmes :

Feuille de fève

Haut poussée

Comment le sommeil peut-il venir

À qui n'a qu'un seul fils.

(Mammeri, 2005, t. II, 135)

Meurtrie, la femme ne savait comment régler ce différend qui, en apparence, ne la concernait pas. Elle prit cependant sur elle de fournir la réponse adéquate, car la parole est ici l'équivalent d'une arme. Elle alla chercher du secours auprès du Cheikh Mohand (voir supra) qui lui recommanda de dire ceci :

Feuille de fève

Haut poussée

Ils sont dix c'est pour détruire

Il est seul et il bâtit.

(ibid., 136)

La seule manière de se défendre consistait à trouver quatre vers significatifs qui pouvaient détruire les assertions de ses adversaires. La réponse est construite sur le même mode, c'est une offensive qui ne dit pas son nom, qui peut être chantée ou dite. Elle doit respecter le fond et la forme.

L'adresse indirecte est notamment à l'œuvre dans la coutume berbère (que l'on retrouve à l'échelle nord-africaine) qui interdit aux époux de s'interpeller directement et de s'appeler par leur prénom. On passe souvent par une tierce personne (parler au fils ou à la fille pour qu'ils s'adressent soit à leur père soit à leur mère, selon l'émetteur) ou encore par les jeux du langage. 

beaucoup de cultures comme les énigmes (timsal), ou encore les devinettes (timserâaq) dont le but est à la fois ludique et pédagogique : «quelle est la fève que l'on plante et qui ne germe pas ?» : le mort; ou « il regarde sans jamais entrer » : la tuile.

Un homme, disait-on, passait à travers champs avec son âne et sa femme. Il piquait son âne en l'injuriant. Les gens autour ne comprenaient pas les raisons d'un tel comportement, mais la femme, elle, avait saisi qu'elle était la destinataire du message. Elle aussi a entonné un chant en direction de l'âne en lui disant: "Toi au moins tu m'écoutes et tu me conduis, ce n'est pas comme ce maudit voisinage ». Il en est ainsi de tous les messages où prédomine la pudeur.

On raconte dans le village des A. B. qu'un homme ne voulait plus avoir de relations sexuelles avec sa femme depuis qu'elle avait accouché. Il avait peur d'être englouti par le vagin de la jeune femme, car il ne s'imaginait pas que le bébé avait emprunté la même voie que celle qui lui avait permis de pénétrer sa femme. La mère inquiète se demandait comment faire passer le message à son benêt de fils. Un bon matin, elle prépara une galette et lui servit du miel et dit : «Quand le miel couvre toute la surface du plat et il suffit d'y mettre le doigt pour voir qu'il est aisé de faire un trou... mais cette béance n'est que momentanée puisqu'en retirant son doigt les choses se remettent en place... Il en est ainsi des femmes, elles sont à l'image du miel... » Par cette métaphore, la mère put exprimer l'indicible dans le langage courant à son fils qui à son tour en fit part à ses amis qui lui expliquèrent que c'était là une réponse à sa phobie des relations sexuelles post-partum.

Il en est de même de cet homme (toujours dans le village des A. B.) qui traversait le village. Tout à coup, il aperçut une silhouette féminine courbée en train de bêcher. La tradition stricte n'autorise pas les hommes à saluer les femmes. Mais le passant trouva un moyen d'exprimer haut et fort son opinion sur la femme en s'adressant en vers à son âne :

Sauvons-nous d'ici

Il n'y a rien à gratter (en kabyle à croquer parce que la femme est vieille)

Immédiatement la femme répondit :

Madame n'est rien d'autre

Qu'un raisin sec fripé (entendre qu'elle est aussi douce qu'un raisin. La douceur

prime sur les plis - litt. rides - du raisin)

Et à l'homme de répondre :

Puisque c'est ainsi, je jure par Dieu

Que j'irai vêtu (entendre qu'ils vont s'accoupler sans avoir à se déshabiller).

Ce jeu de langage est très courant en littérature orale. Il y a des genres qui regorgent de situations où l'adresse indirecte est de mise. Cela permet de tisser des liens avec autrui sans jamais avoir à le dire publiquement ni même directement à l'intéressé.

\section{Genres littéraires}

Des genres littéraires où l'implicite prédomine sont largement connus, car communs à Les contes d'animaux (le chacal, le hérisson où la ruse est purement verbal) regorgent également d'exemples ou lmaâna a valeur de schème structurant. Un large pan de la littérature orale kabyle et berbère est consacré à tiherci (la ruse positive) qui n'a pas seulement pour valeur le jeu, mais a pour fonction de déterminer des comportements sociaux. J'ai consacré un ouvrage à la ruse du chacal et à la manière avec laquelle les 
agents sociaux s'approprient ses qualités pour se situer dans l'échiquier social et politique (Yacine, 2001).

Hormis les fables ou les contes d'animaux, l'adresse indirecte est également présente dans le conte en général comme celui de la fille et du roi où le lecteur est à même de constater comment le pouvoir peut être réductible à une simple performance langagière et ainsi inverser les rapports de force: le dominant peut être battu symboliquement par son dominé parce qu'il ne saisit pas le sens second des mots. C'est ainsi qu'une fille jeune, chétive et pauvre arrive à avoir raison de son époux et roi parce qu'elle a la maîtrise de la langue en usant de l'adresse indirecte (Yacine, 2007).

Mais le code distingue deux types d'adresse indirecte: celle qu'utilisent les dominés (comme ici les femmes ou comme le Hérisson dans les contes animaliers) et celle dont vont user ostensiblement les maîtres du verbe parce que dotés d'un statut charismatique exceptionnel. Ces statuts ne sont pas figés, ils sont amenés à évoluer en fonction des situations et des rapports de force.

\section{Poésie chantée}

Il est également admis que les grands poètes, les devins renommés ont un langage châtié et emploient des paraboles que seuls les initiés peuvent décoder. Mais la langue à elle seule ne suffit pas. Elle doit être habillée, c'est-à-dire avoir un support : rythme, musicalité, etc.

Le genre poétique le plus prisé pour ce type de compétitions linguistiques s'appelle izli (pl. izlan) commun à l'ensemble du monde berbère. Ce sont de courts poèmes chantés dont la base est constituée de deux distiques consacrés généralement à la poésie amoureuse que s'échangent (sous forme de joute: elle et lui) les jeunes amoureux. Ces poèmes peuvent être également chantés par les jeunes garçons, les bergers et les femmes dans des circonstances bien déterminées par le calendrier social (fêtes, mariages, circoncision, réunions dues à un événement spécifique). L'izli s'oppose à la grande poésie d'auteur (taqsit), qui est le fait des hommes et est consacrée aux valeurs sociales, politiques et aux questions religieuses et surtout destinée à être déclamée en public. L'izli est produit soit à l'intérieur des maisons (afrag, enclos mis pour la cour intérieure), soit dans les champs ou encore sur la place publique envahie par les jeunes au moment où les plus âgés la désertent. L'izli est tourné vers le monde de l'intériorité, des affects et de la féminité. Il sert de banque de données aux femmes pour puiser les paroles adéquates, soit pour régler des comptes, soit pour nouer des relations amoureuses. En fait, la poésie en général (et l'izli en particulier) constitue un formidable médium permettant de jouer sur les doubles sens et sens cachés des mots, car l'amour est officiellement tabou.

Aussi le groupe dominant s'est-il ingénié à inventer des canaux permettant de gérer la libido tout en préservant l'intégrité et l'ordre, d'où l'existence des urar-s (litt. jeux) qui constituent, dans la pratique, une soupape de sécurité où le dire sans dire prend toute sa place. Les femmes se réunissent lors de ces séances d'urar-s et s'adonnent à la production de poésies chantées. Elles sont plusieurs (cent voire deux cents), elles chantent et miment des scènes qui renvoient à la copulation. Lors de ces cérémonies, elles créent et transmettent de la poésie. En été, le rite se déroule dans une cour intérieure (afrag) divisée en deux parties par une simple couverture qui les sépare visuellement des hommes, mais les rapproche d'eux au niveau de l'ouïe. Les hommes 
sont à l'écoute des propos féminins et de tout ce qui peut les délivrer du poids du nondit les oppressant l'année durant. Elles composent des joutes provocantes où elles les narguent ouvertement en entonnant des chants les plus osés de la mémoire collective. Les hommes se trouvant de l'autre côté de la couverture font mine de ne rien entendre, mais ils savent, au fond d'eux, qu'ils sont les destinataires de ces cadeaux symboliques. Grâce à l'adresse indirecte, les femmes peuvent ainsi s'adonner à une libre expression, ce que le face-à-face (qabel) interdit absolument. Proférées en dehors du rite et sans support poétique et/ou musical, ces mêmes paroles peuvent être sujettes à scandale voire à meurtre. Ainsi, chacun à leur manière, dominants et dominés jouent sur l'ambiguïté et l'implicite que procure l'adresse indirecte et qui constitue une véritable courroie de transmission dans le respect des règles sociales (Yacine, 1988).

\section{La langue de l'autre: un détour pour se dire}

Le discours amoureux - tabou dans une société patrilinéaire où la sexualité est niée - va également trouver dans la poésie, dans la musique, dans la traduction, la création en langue étrangère, une véritable soupape de sécurité. Dans la Kabylie des années soixante, il était mal vu d'écouter des chansons d'amour émises par la chaîne kabyle. Mais ce qui était interdit dans la langue maternelle était en revanche possible si la même chanson était produite en arabe. On peut comprendre pourquoi les chanteurs chaâbi (genre connu à Alger dans les années 1920) ne chantaient pas en kabyle (la plupart étaient pourtant kabyles), mais en arabe. La langue arabe leur ouvrait un champ d'expression beaucoup plus large et contribuait à les libérer du tabou. On retrouve le même phénomène avec l'homosexualité qui, pour se dire, s'exile dans la langue de Molière comme l'illustre actuellement le jeune écrivain marocain Taïa. Dans une interview télévisée, il déclarait sans ambages qu'il ne pouvait dire dans sa langue (et à sa mère) qu'il était homosexuel. Le passage par le français (l'écrire d'abord et le déclarer ensuite) rendait le tabou acceptable. Il avait ainsi réussi à transmettre le message à sa famille via la langue de l'autre et en différé. C'est assurément ce qui s'est produit pour la visibilité de tout un pan de culture orale kabyle qui traitait des sentiments.

\section{De la traduction à l'écriture}

Par le passé, on pouvait retrouver des traces de cette littérature dans la traduction, qui permettait ainsi de contourner la langue maternelle (Amrouche, 1939). Les premiers francophones de culture berbère vont utiliser la langue française en adresse indirecte, comme un biais (bias) pour s'exprimer et exprimer les tabous du monde social colonisé en passant par la société coloniale. On peut dès lors s'interroger sur le sens profond que revêt la langue de l'autre (dans des sociétés à tradition orale) vécue comme expérience favorisant une véritable catharsis. N'est-elle pas ce substitut de leur propre parole, une parole interdite qui passe par des codes étrangers pour enfin revenir à la culture d'origine ? La poésie d'amour - hors des canaux traditionnels de transmission - a été sauvée de l'oubli grâce à ce formidable médium qu'a été la traduction française (et la transcription qui la sous-tend). La langue française atténuait en quelque sorte la charge " érotique " dont la langue maternelle était investie. "Ce que je ne pouvais en aucun cas dire dans ma langue maternelle, je l'ai fait en français ", déclare une jeune femme 
enquêtant sur la tradition orale. À travers son travail de recherche, elle s'adressait indirectement aux siens.

Des auteurs que nous avons étudiés et qui ont baigné dans au moins deux cultures (berbère, française ou berbère, arabe, française) adopteront cette posture à l'égard de l'autre sans jamais avoir à ôter le masque. Si, pour les hommes (Amrouche, Feraoun, Mammeri, Kheir-Eddine), il s'agit d'entreprendre une lutte au niveau politique - parce qu'affronter directement (qabel) l'autre s'avère impossible -, ce sera en revanche différent pour les femmes, leur lutte étant un combat pour une affirmation identitaire et sexuelle.

41 La tradition musulmane d'Afrique du Nord, comme il a été dit, ne permettait pas aux femmes d'aller à l'école ${ }^{3}$. Les écoles traditionnelles (zaouia, timaâmmart) destinées à former des « savants » religieux étaient fermées aux femmes sauf exception ${ }^{4}$. L'écriture va fonctionner comme une adresse indirecte, car elle permet aux femmes de décrire et de dénoncer des pratiques oppressives émanant de leur propre société : la sexualité, le rapport au corps, les affects, la place de l'individu en société.

\section{De l'écriture à la prise de parole}

42 Le genre de prédilection favorisant cette découverte de soi est représenté par le journal intime $e^{5}$. Dans le champ littéraire nord-africain et plus particulièrement des Berbères, ce genre est très peu familier, à l'exception de Jean Amrouche (1906-1962), de Taos Amrouche (1913-1975) et de Mouloud Feraoun (1913-1962). On peut comprendre les raisons de ce choix, sans doute le fait de cette double domination culturelle et linguistique qui a fonctionné comme un moyen indirect de s'exprimer, en livrant au lecteur étranger un poids imprimé par maintes souffrances interdites à l'expression, dans des situations dictées par une norme stricte.

43 En Afrique du Nord, la langue française importée (imposée) par la colonisation a donc été, sur le plan de l'expression en général et des affects en particulier, une forme de libération, parce qu'elle a été vécue comme un moyen double d'émancipation de la société dominante (coloniale) et de la société dominée (colonisée) en raison de ses lois et de ses tabous ${ }^{6}$.

Pour échapper à la confrontation avec la langue maternelle, l'écriture se sert de la langue de l'autre, donc en adresse indirecte, puisque le public concerné en prend connaissance grâce à cette langue. Le recours à la langue de l'autre facilite l'accès à un champ des possibles jusque-là impossible et réintroduit le marginal dans la normalité. Parce qu'interdites d'expression sur la scène publique, les femmes vont se saisir de ce moyen pour occuper un espace aussi réduit soit-il.

Nous nous fonderons sur la quête de soi chez Taos Amrouche qui figure parmi les premières femmes à avoir osé lever le voile de l'intimité grâce à l'écriture en langue française, ce qu'assurément la sienne interdisait. La langue de l'autre a permis à la toute première génération de quitter le giron familial et, du coup, l'univers féminin ; un univers où, dans l'esprit des petites colonisées, où tout était perçu négativement en raison de l'imposition de schèmes de vision impliquant une division du monde et de ses pratiques. Les femmes étaient non seulement privées de culture "savante ", mais de plus elles évoluaient dans un espace réduit (la maison, en ville ; le hameau et le village, à la campagne) aux horizons bouchés. Dans les années 2000, une enquête sociologique menée auprès d'une vingtaine de femmes (toutes issues de groupes linguistiquement 
minorés d'origine algérienne $)^{7}$ montre précisément ce hiatus qui existe entre le monde des mères et celui de leurs filles transformées par l'école. Ce n'est pas seulement l'espace qui est problématique: il y a souvent la langue, la culture de l'extérieur (différente de celle de l'intérieur, de la famille) et les règles du monde de la rue et de ce qui est supposé représenter la culture dominante.

Pour beaucoup d'intellectuelles, il s'agissait de régler un contentieux avec le groupe. Selon qu'elles étaient en parfait accord ou non avec leur mère, l'idée dite ou non dite consistait à opérer une rupture. L'école offrait un moyen incontestable de transcender symboliquement la situation offerte à leur semblable dans le contexte traditionnel (colonisé ou non). Plus que les citadines, les auteures issues de la montagne ont eu très souvent le sentiment que la culture de l'autre était une forme de "sortie» de leur univers, qui leur permettait d'exister en tant qu'individus.

Pour certaines, la langue française était un instrument de libération et la fiction une libération encore plus grande puisqu'elle permettait en fait de dire sans dire, d'écrire sur soi, mais en s'adressant à un auditoire autre (francophone avec qui on partage des valeurs communes) susceptible de lire et de comprendre. La langue étrangère (parce qu'elle est extérieure à soi et au groupe), justifient-elles, est garante d'objectivité, elle devient par la force des choses et de l'histoire le révélateur de soi, de son identité, de sa culture et de son genre ${ }^{8}$. Se saisir du français, c'est se situer dans un autre monde, prendre de la distance y compris par rapport à soi-même à «sa culture, son appartenance à un groupe déterminé, sa sexualité », confirme Aldjia.

L'écriture est ainsi une échappatoire, une confession qui leur permet de se dire sans se trahir, car elles sont dans l'univers de l'autre, dans son espace, dans ses codes culturels. Substitut de leur propre langue, le français ne servait qu'à libérer la parole berbère ou arabe interdite d'expression.

\section{De la prise de parole à la rupture d'un pacte}

Aussi paradoxal que cela puisse paraitre, l'exemple de Nouara (Yacine, 1995), cette poétesse kabyle qui a émigré dans les années soixante en France, est à la fois semblable et différent des précédents. Semblable dans la mesure où l'écriture est vécue comme une double émancipation : à la fois réelle et symbolique, puisque Nouara se sert de la plume pour inverser les rapports de force. Radicalement différent parce qu'elle ne savait ni lire ni écrire dans aucune langue, et elle se sert de sa langue maternelle pour dire par écrit ce que jusque-là il lui était impossible d'exprimer oralement. Ce tour de force, elle le réussit grâce à l'émigration qu'elle vit comme un exil. C'est précisément cette situation d'épouse exilée de son pays et n'ayant pas été scolarisée qui va contribuer dans un premier temps à une dépression avant qu'elle ne la transforme en création.

50 L'apprentissage de l'alphabet et de la langue française (dans la banlieue lyonnaise des années 1960) lui a permis de transcrire ses poèmes. La maîtrise de l'alphabet a été vécue comme une véritable libération de la tradition et une sortie du mutisme et de l'autisme dans lesquels elle était enfermée. Cette capacité de saisir le stylo l'autorise à dire l'indicible en se vengeant de la société et en rejetant ses règles. Nouara voulut témoigner de sa souffrance, l'écrire c'était, pour elle, partager la tragédie dans laquelle elle était enfermée (orpheline, plusieurs fois divorcée, veuve et sans enfants) la révéler au monde. Comment y parvenir si ce n'est en se débarrassant de cette maudite intimité 
(Yacine, 1995)? Elle doit se déshabiller, se mettre à nu et permettre aux lecteurs d'entrer dans son monde. Le fait de transcrire l'autorise à dévoiler des sentiments jusque-là tabous.

51 Le fait d'écrire participerait ainsi de cette rupture d'un pacte social dans lequel le créateur ne se sent plus partie prenante. Dans le cas présent, Nouara est bien différente de la génération précédente (comme si la langue berbère en passant de l'oral à l'écrit était désaffectivée), elle réussit ce tour de force dans sa propre langue, ce qui malgré tout reste très préjudiciable pour elle. Mais elle semble ne pas en avoir tout à fait conscience... Écrire, ce n'est pas affronter (qabel); c'est s'adresser à l'autre sans le lui dire.

Écrire sur soi, c'est larguer les amarres, rompre le pacte qui vous lie aux autres... C'est parler, transgresser le code (le formel), dire de façon indirecte même si l'indirect est vite compris de tous, mais qu'importe, il est reconnu comme code faisant lien.

Dans le village, la famille, on joue toujours le jeu, on fait semblant et on doit accepter les règles énoncées de façon explicite ou implicite... Le passage à l'écrit est un acte important, car on écrit sur soi, certes, mais en impliquant les autres tout en faisant mine de s'en détacher. Il est faux de croire qu'il ne s'agit que d'une personne, c'est toute la société qui est concernée, ses codes, ses réalités, mais aussi ses phantasmes... Si toutes les femmes décrivaient de l'intérieur leur propre vécu et celui de leurs proches, il se produirait une véritable révolution sociale (Méchakra, 1979). L'expression féminine est importante quelle que soit la forme qu'elle prend, car elle est à l'origine d'un bouleversement des valeurs, comme on peut le voir dans les propos suivants (recueillis lors de mon enquête citée plus haut).
Ma mère n'a pas voulu de moi... je suis arrivée sans crier gare et, de plus, j'étais une fille. Elle me répétait toujours : pourquoi n'es-tu pas un garçon? Ah! Si cette tête dure, cette tête de mule était seulement un garçon!
J'ai pris mon mal en patience et toute petite je voulais lui prouver que j'étais mieux qu'un garçon: j'étais la première du village à avoir fait des études et l'une des premières femmes de mon pays à faire ce que je fais. J'avais réussi à relever le défi et à montrer à ma mère ce que je valais grâce à la langue française que je mettais ostensiblement entre elle et moi tout en sachant que je m'en servais aussi comme détour pour revenir à moi-même. Mais intérieurement j'en avais souffert, j'ai dû éliminer en moi tout ce qui était féminin : à cause d'elle je m'étais transformée en homme, par mon métier, mais aussi par mon attitude. Je détestais les tâches féminines, je détestais les chiffons, les bavardages, bref, ce qui pouvait me rappeler ma mère. Indirectement, j'avais répondu à ma mère sans avoir à être désagréable ni méchante avec elle.

Cette autre a tenu à poursuivre des études supérieures et à devenir chercheure parce que son mari se moquait d'elle à chaque fois qu'elle prenait un livre : "Tu vas devenir le docteur Untel! Ce serait le monde à l'envers!» La position dans laquelle elle se trouvait ne lui permettait pas de répondre sur-le-champ, mais de lui montrer plus tard, sans avoir à le dire, ce qu'elle savait faire lorsqu'elle serait en mesure de devenir un des meilleurs chercheurs du pays. La recherche sur les femmes - s'approprier leur parole a en quelque sorte servi à faire comprendre indirectement à son époux ce qu'elle était en mesure de faire sans jamais avoir à le lui dire et, du coup, à comprendre les créations millénaires des femmes illettrées célébrant la sexualité sur un mode biaisé qu'est l'adresse indirecte.

Il nous a paru important de déconstruire la perception de la parole dans le sens figé qu'on lui attribue et de nous interroger sur ce que représente l'adresse indirecte en 
dehors du jeu des métaphores, de l'initiation à la maîtrise de la langue et de la culture orales. La parole dépasse largement la fonction que lui attribue le sens commun. À l'origine du lien social, elle peut donner la vie comme elle peut donner la mort (ainsi du code de l'honneur). La parole prédispose ainsi l'agent à exercer (ou à subir) un rapport de force. Cependant, lorsque les règles du jeu ne permettent pas l'exercice légitime de la violence (pour parler comme Weber), le groupe offre d'autres issues pour affronter, détourner, contourner un pouvoir en s'appuyant sur d'autres registres.

Mais plus que cela, l'adresse indirecte renvoie à la structure sociale et politique et révèle, du coup, les modes de gestion des discours en rapport avec le statut des individus. Plus loin encore, c'est le sens profond qu'elle peut revêtir sans que les agents sociaux en aient pleinement conscience comme on peut le constater dans la mémoire esthétique relative à la poésie chantée et à la dimension symbolique que recèle la mémoire. C'est sans doute grâce à la mémoire que l'adresse indirecte resurgit sous d'autres formes - et c'est de cette dernière parce qu'elle est incorporée - dont les femmes se saisissent pour s'exprimer.

\section{BIBLIOGRAPHIE}

ALLIOUI, Youcef, 1990, Timsal. Énigmes berbères, Paris, L'Harmattan.

AMrouche, Jean, 1939, Chants berbères de Kabylie, Tunis, Monomotapa.

AMROUCHE, Jean, 2008, Journal 1928-1962, Éditions Non Lieu.

AMrouche, Taos, 1975, L'Amant imaginaire, Paris, Morel.

BOURDIEU, Pierre, 1980, Le Sens pratique, Paris, Éditions de Minuit.

FINLEY, Moses, 2002, Le Monde d'Ulysse, Paris, Seuil [1 $1^{\text {st }}$ ed., The World of Odysseus, 1954].

GENEVOIS, Henri (Père), 1963, 350 énigmes kabyles, Fort nationales, Fichier de Documentation

berbère.

JOUAD, Hassan, 1989, Le langage de Lmaâna, L'esthétique de l'implicite, Études et documents berbères, $\mathrm{n}^{\circ} 6$, p. $158-168$.

MAMMERI, Mouloud, 1969, Les Isefras, Paris, Maspéro.

MAMMERI, Mouloud, 1978, Dialogue sur la poésie orale, Actes de la recherche en sciences sociales, $\mathrm{n}^{\circ} 23$, p. 51-66.

MAMMERI, Mouloud, 1980, Poèmes kabyles anciens, Paris, La Découverte.

MAMMERI, Mouloud, 2005, Cheikh Mohand a dit, t. II, traductions Alger, CNPH.

MECHAKRA, Yamina, 1979, La Grotte éclatée, Alger, SNED.

YACINE, Tassadit, 1988, L'izli ou l'amour chanté en kabyle, Paris, Éditions de la Maison des Sciences de l'Homme. 
YACINE, Tassadit, 1992, Anthropologie de la peur : l'exemple des rapports hommes/femmes, in Tassadit Yacine (dir.), Amour, phantasmes et sociétés en Afrique du Nord et au Sahara, [Actes du colloque international des 14-15-16 juin 1989], Paris, L'Harmattan, Awal, p. 3-27.

YACINE, Tassadit, 1995, Piège ou le combat d'une femme algérienne, Paris, Publisud.

YACINE, Tassadit, 2001, Chacal ou la ruse des dominés, à l'origine du malaise des intellectuels algériens, Paris, La Découverte.

YACINE, Tassadit, 2007, Servir les hommes ou L'art de la domination déniée, Agone, [La joie de servir], $\mathrm{n}^{\circ} 37$, p. 53-69

YACINE, Tassadit, 2009, Taos Amrouche ou l'entreprise d'objectivation de soi, Awal, Cahiers d'Études berbères [Taos Amrouche, une féministe avant l'heure ?], nº 39, p. 7-23.

\section{NOTES}

1. Cela nous rapproche du demiurgos, du monde méditerranéen ancien, cf. Finley (2002).

2. Il en est ainsi du célèbre sage Bou'Amrane connu dans l'ensemble du monde berbère.

3. On a pu observer dans certaines familles lettrées en Kabylie, dans l'Anti-Atlas, des écoles à domicile destinées aux seules femmes.

4. Avec la conquête coloniale (1830) et l'instauration de l'école laïque (1883), de nombreux enfants issus de milieux modestes (ceux vivant dans les montagnes) vont accéder à l'éducation et ainsi s'approprier la culture de l'autre pour des raisons économiques et sociales d'abord, culturelles ensuite. Les filles qui fréquentent l'école sont rares dans les années 1930, elles y accéderont progressivement.

5. La sociologie et la littérature ont toujours classé le roman psychologique (familial) du côté de la féminité. Il en est ainsi pour le journal intime même s'il arrive à des auteurs masculins de se consacrer à ce genre d'écrits. Peut-être se sentaient-ils dominés, à l'instar des femmes.

6. Tout en accordant aux hommes la possibilité d'étudier, de travailler à l'extérieur, il n'en demeure pas moins que le groupe exerce son autorité (voire son diktat) parce qu'ils sont perçus comme ses fidèles représentants destinés à assurer la préservation de son code traditionnel, de ses coutumes.

7. Enquête conduite par moi-même auprès de femmes en Afrique du Nord et en France.

8. «Je n'étais pas comme les autres, j'étais un cas à part. Le regard d'autrui a fini par déteindre sur moi. J'appartiens à une espèce humaine bizarre... Jeune, j'en avais souffert, mais plus âgée et en prenant du recul, j'ai regardé cette femme (moi perçue dans la distance) avec un regard éloigné tenant compte du temps écoulé, mais aussi du lieu (le lieu n'est pas étranger à ce qui nous arrive). Entre la Tunisie et la France, il y a en réalité des milliers de choses. La distance mise entre Tunis et Paris m'a permis de comprendre que mon histoire était à dire, mais comment ? Dans quelle langue ? Le seul moyen était de passer par la langue de l'autre. » 


\section{RÉSUMÉS}

La société kabyle est connue pour ses différentes formes de productions orales (poésies, chansons, proverbes, devinettes et autres discours versifiés) dont certaines ont fait l'objet de traduction et d'analyse. Il s'agit très souvent de textes en relation avec l'adresse directe. Dans cet article, Tassadit Yacine s'attache à décrire la place de l'adresse indirecte dans différents espaces d'expression: dans la vie quotidienne d'autrefois mais aussi dans la prophétie, comme dans l'univers de la poésie chantée. L'adresse indirecte est ce mode d'expression privilégié des hommes et des femmes issus de la tradition, mais aussi des intellectuelles francophones qui en font un moyen privilégié de dire dans la langue française ce qu'elles ne s'autorisent pas à dire dans leur langue maternelle.

Kabyle society is known for its various forms of oral (poetry, songs, proverbs, riddles and other speeches in verse), some of which have been translated and analyzed. It is very often texts in connection with the direct address. In this article, Tassadit Yacine attempts to describe the role of indirect address in different areas of expression in everyday life of the past but also in prophecy, as in the world of sung poetry. The indirect address is the mode of expression for men and women from the intellectual tradition for educated francophone who use French to say things that would not be permitted in their own language

\section{INDEX}

Keywords : Indirect Address, Women, Language, Written Production, Dominated, Translation, Kabyle people, Kabylie, Anthropology

Thèmes : anthropologie (Afrique)

nomsmotscles Kabyles

Index géographique : Kabylie

Mots-clés : adresse indirecte, femmes, langue, production écrite, dominés, traduction 